\title{
Development and Psychometric Evaluation of the Utrecht Grief Rumination Scale
}

\author{
Maarten C. Eisma • Margaret S. Stroebe - Henk A. W. Schut • \\ Jan van den Bout • Paul A. Boelen • Wolfgang Stroebe
}

Published online: 23 August 2013

(C) Springer Science+Business Media New York 2013

\begin{abstract}
Two different types of ruminative coping, depressive rumination and grief rumination, negatively influence bereavement outcome. Although grief-specific rumination is likely to be relevant in the bereavement context no internationally validated scale to measure grief rumination exists. Therefore, the current contribution aims to validate the Utrecht Grief Rumination Scale (UGRS), a scale developed to measure grief-specific rumination, in an English sample. Psychometric properties of the English UGRS were compared with those in a Dutch sample. 204 British adults ( $89 \%$ women, $11 \%$ men), bereaved on average 16 months ago, and 316 Dutch adults ( $88 \%$ women, $12 \%$ men), bereaved on average 12 months ago, filled out online questionnaires. Different types of rumination (grief rumination, brooding, reflection, trait rumination) and symptoms of psychopathology (anxiety, depression, complicated grief) were measured. A correlated five factor model provided the best fit for the UGRS. Multi-group comparisons showed that the factor structures of the English and Dutch version of the UGRS were highly similar across language groups. The UGRS showed excellent reliability. Results further supported the construct, convergent, divergent and concurrent
\end{abstract}

This research was supported with a Zon-Mw TOP grant of the Dutch Association for Scientific Research (NWO).

Electronic supplementary material The online version of this article (doi:10.1007/s10862-013-9377-y) contains supplementary material, which is available to authorized users.

M. C. Eisma $(\bowtie) \cdot$ M. S. Stroebe $\cdot$ H. A. W. Schut

J. van den Bout $\cdot$ P. A. Boelen

Department of Clinical and Health Psychology, Utrecht University,

Heidelberglaan 1, 3584 CS Utrecht, Netherlands

e-mail:m.c.eisma@uu.nl

W. Stroebe

Department of Social and Organizational Psychology,

Utrecht University, Utrecht, Netherlands validity of the English UGRS. The psychometric properties of the Dutch UGRS corroborated these findings. The UGRS appears a valid instrument to assess grief-specific rumination in international research and may have potential applicability as a clinical instrument to assess rumination in individuals with problematic grief.

Keywords Rumination - Scale - Questionnaire - Validation · Grief $\cdot$ Repetitive thought

Repetitive thought has been a core tenant of theories on adjustment to bereavement for nearly a century. As early as 1917, Freud observed that bereaved individuals often think recurrently about the deceased person, the loss experience and the changed world in which they now live (Freud 1917/1957). He believed this thought process to be a crucial component of grief work, an adaptive process whereby individuals gradually come to terms with a loss by repeatedly confronting themselves with this life-changing event. This idea has echoed through bereavement literature since that time (Bowlby 1980; Lindemann 1944; Stroebe 1992).

Recently, however, increasing attention has been devoted to a maladaptive thought process after the death of a loved one, rumination. Rumination is broadly defined as repetitive and recurrent, self-focused negative thinking about past negative experiences and/or negative mood (Michael et al. 2007). Two different types of ruminative thinking, depressive rumination and grief rumination, have been proposed as playing an important role in the development and persistence of depression (Nolen-Hoeksema 2001) and complicated grief (Boelen et al. 2006).

First, depressive rumination, repetitively and passively focusing on depressive symptoms and on the possible causes and consequences of these symptoms (Nolen-Hoeksema et al. 2008), may be a risk factor after loss. Depressive rumination 
has been proposed to be maladaptive, because it increases accessibility of negative thoughts and memories, impairs problem solving, interferes with instrumental behavior, and drives away social support (for a review: Nolen-Hoeksema 2001). Indeed, it is associated with, and prospectively predicts depressive symptoms following bereavement (e.g. Nolen-Hoeksema et al. 1994, 1997).

While depressive rumination may thus play a role in the aftermath of a loss, a related, but fundamentally different phenomenon, namely grief rumination, has also been linked to negative consequences after the loss of a significant other. Grief rumination has been characterized as repetitive and recurrent thinking about the loss, its consequences and/or negative emotions related to the loss (Boelen et al. 2003a, b, 2006; Boelen and van den Hout 2008; van der Houwen et al. 2010). Thus, whereas depressive rumination is focused exclusively on depressive feelings and symptoms, grief rumination consists primarily of thoughts about the reasons for and meaning of the loss and general loss-related emotions. Previous research has shown that grief rumination is related to severity of depressive and complicated grief symptoms (Boelen et al. 2003a, b; Boelen and van den Hout 2008). Moreover, the relationship between personality characteristics of recently bereaved individuals, such as neuroticism and attachment style, and symptoms of depression and complicated grief, were found to be mediated by grief rumination in a longitudinal study (van der Houwen et al. 2010). While these findings underline the importance of grief-specific ruminative thought in determining the consequences of the loss of a loved one, no reliable and valid measure of grief rumination has yet been developed for international scientific research.

This is surprising, because there are compelling theoretical reasons why grief rumination is different from and more likely to occur after a loss-experience, than depressive rumination. First, the emotions bereaved individuals ruminate about are likely to include more than just depressive emotions. Although sadness is a common reaction to the death of a significant other, and a depressive disorder can develop as a consequence of bereavement, mourners commonly report many different emotional experiences, such as dysphoria, anger and anxiety (for a review: Stroebe et al. 2007), all of which can be the focus of rumination (Robinson and Alloy 2003; Sukhodolsky et al. 2001). Therefore, ruminative thinking after bereavement is likely to be focused on general lossrelated negative feelings rather than exclusively on depressive emotions (cf. Nolen-Hoeksema 2001).

The second reason why grief rumination is more likely to occur after a loss-experience than depressive rumination, is that a loss logically induces different types of ruminative thoughts than a dysphoric or depressive episode unprecedented by a loss. Rumination has been characterized as a discrepancy-focused thinking style, which revolves around a common instrumental theme (Martin and Tesser 1996).
Depressed individuals frequently report that they ruminate to try to understand their depressive feelings (Papageorgiou and Wells 2003) and frequently-used measures of depressive rumination, such as the Ruminative Response Scale, or RRS (Nolen-Hoeksema and Morrow 1991; Treynor et al. 2003) largely consist of items that measure thoughts about possible, but unclear causes of these feelings. Thus, in response to a discrepancy in mood state (one feels depressed when one wants to feel happy) a depressed ruminator repeatedly analyzes the ambiguous precedents of his current low mood. However, the source of negative feelings is less equivocal after a loss; negative emotions experienced by bereaved individuals are often intrinsically linked with the death of a loved one. Therefore, a bereaved person may attempt to resolve a discrepancy in mood state and make sense of what has happened by recurrently thinking about the causes and consequences of the death (Tait and Silver 1989). Similar to traumarelated rumination (Michael et al. 2007) then, rumination following loss is focused more strongly on the life-changing event (i.e. the death of a loved one), than on the unclear causes of depressive feelings and symptoms.

Since there are both theoretical and empirical arguments for continued investigation of the effects and correlates of griefspecific rumination, the primary goal in this study was to validate a new English version the Utrecht Grief Rumination Scale (UGRS), a recently developed instrument to assess different facets of ruminative thinking after bereavement. To accomplish this goal, the psychometric properties of UGRS in an English sample were compared with those of the UGRS in a Dutch sample, in order to investigate the comparability of both measures. We thereby aimed at corroborating and extending the results of a preliminary study which examined the psychometric properties of the Dutch UGRS (Eisma et al. 2012).

In the above mentioned study, the construction of the UGRS was accomplished in two steps. First, 30 items were constructed on the basis of literature on depressive rumination (NolenHoeksema and Morrow 1991; Treynor et al. 2003), traumarelevant rumination (Michael et al. 2007) and grief-relevant rumination (e.g., Boelen et al. 2003a, b, 2006; Boelen and van den Hout 2008; van der Houwen et al. 2010), as well as semi structured interviews with bereaved individuals and clinical observations of professional grief therapists. These 30 items were designed to tap five themes of grief-specific ruminative thought: analysis of (emotional) reactions to the loss (cf. NolenHoeksema 2001; Treynor et al. 2003; van der Houwen et al. 2010), thoughts about the consequences of these reactions (cf. Nolen-Hoeksema 2001; Nolen-Hoeksema and Morrow 1991), thoughts about the injustice of the death (including "why questions") (cf. Boelen et al. 2006; Michael et al. 2007; van der Houwen et al. 2010), thoughts about the meaning and consequences of the loss, including the reactions of others (cf. Michael and Snyder 2005) and counterfactual thinking about the loss (cf. Boelen et al. 2006; Michael et al. 2007). 
Second, the 30-item UGRS was completed by a sample of 302 recently bereaved Dutch individuals. Using principal component analysis (PCA) a correlated five factor solution was found to best explain the scores on the 30-item UGRS. Next, the three highest loading items on each factor were retained, which resulted in a final 15 -item scale. Finally, a second PCA showed that scores on each of the five subscales all loaded high on a single factor, suggesting that the UGRS may have a hierarchical factor structure. Analyses further demonstrated that the final UGRS exhibited good internal consistency and adequate temporal stability. Convergent and divergent validity was supported by strong positive associations between the UGRS and maladaptive types of ruminative thinking, such as brooding, and weaker relationships with potentially adaptive types of ruminative thinking, such as reflection (Treynor et al. 2003). In support of the discriminant validity, the UGRS could distinguish groups who generally report more severe grief trajectories, such as people who experienced a sudden loss or child loss, from people with less severe grief trajectories. Predictive validity of the UGRS was also supported: in a group of bereaved individuals with initial high complicated grief symptoms, grief rumination was a better predictor of depressive and complicated grief symptom change, when compared to brooding, reflection and rumination measured with the Rumination Reflection Questionnaire (Trapnell and Campbell 1999).

In order to establish the psychometric properties of the English UGRS, we first examined the construct validity by conducting factor analyses of the UGRS in the English sample and a new Dutch sample. Specifically, we compared the fit of a one order factor structure with five correlated factors with the fit of a two order factor structure with five sub-factors and one overarching higher-order factor. Next, we investigated the cross-cultural equivalence of the English and Dutch version of UGRS by conducting multi-group confirmatory factor analyses on the factor model that provided the best fit. Consequently, four types of validity were assessed by testing identical hypotheses in both samples. First, with respect to convergent validity, moderate to large positive correlations were expected between grief rumination and maladaptive types of ruminative thinking, such as brooding (Treynor et al. 2003). Second, with respect to the divergent validity, we predicted associations between grief rumination and adaptive types of ruminative thought (i.e. reflection) would be smaller than those between grief rumination and maladaptive types of ruminative thought (i.e. brooding). Third, with regard to the discriminant validity, grief rumination was hypothesized to distinguish groups that commonly experience more mental health problems after the death of a loved one, such as people who have experienced the death of a child, from groups with less severe grief trajectories, such as people who lost a parent (Stroebe et al. 2007). Fourth, we expect the UGRS to show concurrent validity, that is, grief rumination was hypothesized to predict symptoms of psychopathology over and above sociodemographic and loss-related variables and other types of ruminative thinking, such as brooding and reflection (Treynor et al. 2003) and trait rumination measured with the Rumination Reflection Questionnaire (Trapnell and Campbell 1999).

\section{Method}

\section{Samples}

In both samples, only people who had lost a first-degree relative in the past 3 years were included, to ensure that the participant groups experienced a wide range of symptom and rumination levels. The English sample consisted of 204 British adults ( $11 \%$ male, $89 \%$ female), bereaved on average 16 months ago, with a mean age of $48.5(\mathrm{SD}=13.0)$. The majority of the English participants had lost a partner or parent ( $80 \%$ ), due to natural causes ( $84 \%$ ) and had experienced the loss as unexpected (57\%). The Dutch sample was comprised of 316 adults ( $12 \%$ male, $88 \%$ female), bereaved on average 12 months ago and a mean age of $48.3(\mathrm{SD}=11.9)$. Dutch participants had predominantly lost a partner or parent (81\%), due to natural causes ( $88 \%$ ) and had experienced the loss as unexpected $(47 \%)$. Language groups differed on education level, $\chi^{2}(3)=26.76, p<.01, \omega^{2}=0.23$, and on the relationship with the deceased, $\chi^{2}(3)=11.63, p<.01, \omega^{2}=0.15$. In the English sample, significantly more people indicated secondary school as their highest education and fewer people indicated vocational school was their highest education, when compared to the Dutch sample (i.e., $35 \%$ vs. $27 \%$ and $18 \%$ vs. $34 \%$ ). However, this may be due to differences in the school systems of these countries. In the English sample more participants had lost a parent and fewer participants had lost a partner, in comparison to the Dutch sample (i.e., $42 \%$ vs. $30 \%$ and $38 \%$ vs. $51 \%$ ).

As can be seen in Table 1, scores on rumination and symptoms of psychopathology were higher in the English sample, relative to the Dutch sample, but effect sizes of these differences were small to moderate. These group differences may, in part, be due to the flexible, internet-based recruitment procedure used in this study.

\section{Procedure and Measures}

All participants were recruited over the internet through announcements on websites for online support groups for bereaved individuals or advertisements on the content network of Google in both the United Kingdom and the Netherlands. Interested individuals linked through to a (bilingual) website specifically designed for the current research project, where information was provided about the study. In line with Dutch regulations for scientific research (Ministerie van Volksgezondheid, Welzijn 
Table 1 Means, standard deviations and t-tests of differences on measures of rumination and symptoms in the English and Dutch sample

\begin{tabular}{|c|c|c|c|c|c|c|c|c|}
\hline \multirow[t]{2}{*}{ Scale } & \multicolumn{2}{|c|}{ English } & \multicolumn{2}{|c|}{ Dutch } & \multirow[b]{2}{*}{$t$-value } & \multicolumn{2}{|c|}{$95 \% \mathrm{CI}$} & \multirow{2}{*}{$\begin{array}{l}\text { Cohen's } \\
d\end{array}$} \\
\hline & M & SD & M & SD & & LL & UL & \\
\hline UGRS & 49.4 & 13.6 & 44.3 & 11.7 & $5.10 * *$ & 3.14 & 7.06 & 0.45 \\
\hline RRS brooding & 12.4 & 3.7 & 10.2 & 3.3 & $7.07 * *$ & 1.58 & 2.81 & 0.60 \\
\hline RRS reflection & 11.3 & 3.6 & 10.2 & 3.3 & $3.53 * *$ & 0.48 & 1.71 & 0.30 \\
\hline RRQ rumination & 41.9 & 7.3 & - & - & - & - & - & - \\
\hline HADS anxiety & 12.3 & 4.8 & 10.1 & 4.2 & $5.51 * *$ & 1.41 & 2.98 & 0.48 \\
\hline HADS depression & 10.7 & 5.3 & 9.8 & 4.9 & $1.98 *$ & 0.01 & 1.79 & 0.17 \\
\hline ICG & 67.2 & 26.9 & 55.4 & 23.2 & $5.31 * *$ & 7.43 & 16.16 & 0.47 \\
\hline
\end{tabular}

$U G R S$ Utrecht Grief Rumination Scale, $R R S$ response questionnaire, $R R Q$ rumination reflection questionnaire, HADS hospital anxiety and depression scale, $I C G$ inventory of complicated grief, $L L$ lower limit, $U L$ upper limit

The RRQ was only administered in the English sample

* Significant difference at $p<0.05$

**Significant difference at $p<0.01$

en Sport 2002), people who were interested in participation could access an online questionnaire after reading the research information (e.g., on study goals, confidentiality, advantages and disadvantages of study participation) and giving informed consent.

\section{Sociodemographic Information}

Characteristics of the participant (age, sex and education level) and characteristics of the deceased and the loss (relationship with deceased, time since the loss, cause of death and expectations about the death) were measured with a selfconstructed questionnaire.

\section{Grief Rumination}

The Utrecht Grief Rumination Scale (UGRS) was used to measure grief-specific rumination, defined as recurrent, repetitive and self-focused thoughts about the causes and consequences of the loss and related negative feelings (Eisma et al. 2012). It consists of five subscales of three items each. Participants could indicate how frequently they had experienced certain types of thinking in the past month on a fivepoint scale ranging from 1 (never) to 5 (very often). The subscale Reactions measures thoughts about negative (emotional) reactions to the loss. An example: "(How often in the past month...) did you try to analyze your feelings about this loss precisely?" The subscale Injustice assesses thoughts about the unfairness of the death. An example item is: "(How often in the past month...) did you wonder why this had to happen to you and not someone else?" The subscale Counterfactuals measures counterfactual thoughts about the events leading up to the death. For example: "(How often in the past month...) did you analyze if you could have prevented the death?" The subscale Meaning assesses thoughts about the meaning and consequences of the loss experience. An example item is: "(How often in the past month...) did you analyze what the personal meaning of the loss is for you?" The subscale Relationships measures thoughts related to social support. For example: "(How often in the past month...) did you think about how you would like others to react to your loss?".

The original Dutch UGRS was translated into English by an independent native speaker and then back-translated into Dutch by another independent person fluent in both Dutch and English. Next, the original version was compared to the backtranslated version. Only minor differences existed between these versions and they were addressed by making appropriate adjustments to the English version. The final versions of both scales are added as online supplementary material (Utrecht Grief Rumination Scale.pdf).

\section{Depressive Rumination}

The Ruminative Response Scale (Nolen-Hoeksema and Morrow 1991; Dutch translation by Raes et al. 2009) was used to assess depressive rumination. In the current study, two 5-item subscales of the RRS that show no overlap with depressive symptoms, the 'brooding' and 'reflection' subscale, were used (Treynor et al. 2003). Respondents were asked to indicate how often they exhibit certain behavior if they feel sad, blue or depressed on a four-point scale, ranging from almost 1 (never) to 4 (almost always). An example item of the brooding scale is: "I think: Why do I always react this way?" An example item of the reflection subscale is: "I analyze recent events to understand why I feel depressed." In both 
the English and the Dutch sample the reliability of the brooding $(\alpha=.78 / \alpha=.76)$ and reflection subscale $(\alpha=.78 / \alpha=.78)$ was adequate.

\section{Rumination}

In order to further evaluate the convergent validity of the UGRS in the English sample a second measure of (trait) ruminative thought was included: the rumination subscale of the Rumination Reflection Questionnaire (Trapnell and Campbell 1999). The questionnaire consists of 12 statements for which a participant indicates to what extent these are applicable to them on a five-point Likert scale. An example: "I often reflect on episodes in my life I should no longer concern myself with." The internal consistency of the rumination subscale was good $(\alpha=.86)$.

\section{Symptoms of Anxiety and Depression}

Symptoms of anxiety and depression were measured with the Hospital Anxiety and Depression Scale, or HADS (Zigmond and Snaith 1983) The HADS has been shown to be both a reliable and valid measure of depressive and anxious symptoms in several large Dutch samples, including community samples (Spinhoven et al. 1997). The HADS consists of 14 statements about experiences, of which seven statements tap anxious and seven depressive symptoms. Participants indicated how often or to what extent they have had these experiences in the past week on four-point Likert scales. In both the English sample and the Dutch sample the internal consistency of the anxiety $(\alpha=.86 / \alpha=.86)$ and the depression subscales $(\alpha=.88 / \alpha=.89)$ was good.

\section{Symptoms of Complicated Grief}

Grief complications were measured with the Inventory of Complicated Grief Revised, or ICG-R (Prigerson and Jacobs 2001; Dutch translation by Boelen et al. 2003a, b). The Dutch version consists of 29 and the English version consists of 30 statements about the frequency and intensity of symptoms of complicated grief. Answers are given on a five-point Likert scale ranging from 0 (almost never) to 4 (always). The internal consistency of the ICG was excellent in both the English $(\alpha=.96)$ and the Dutch sample $(\alpha=.95)$.

\section{Results}

\section{Preliminary Analyses}

Before conducting confirmatory factor analyses, the multivariate normality of the data on the UGRS was assessed for both samples on the basis of histograms, QQ-plots and measures of skewness and kurtosis. In the English sample, as for the Dutch, multivariate normality was not violated.

\section{Confirmatory Factor Analyses}

On the basis of principal component analyses in a previous study (Eisma et al. 2012) it was expected that a model with five latent variables (Reactions, Injustice, Counterfactuals, Meaning, Relationships) could best explain the scores on the UGRS in the English sample, and that these findings would be substantiated in the Dutch sample. Moreover, a two-order factor model was considered to be an appropriate solution for the UGRS, since all five factors loaded highly on a single factor (Grief Rumination) in the same study.

The factor structure of both versions of the UGRS was examined through structural equation modeling (AMOS 16.0) using maximum likelihood estimation (Kline 2005). The one order model was tested first. It was hypothesized that three items each would load on each of the five latent variables. The loading of a single item on each factor was constrained to 1.0 to establish a metric for the latent factors. All other factor loadings were freely estimated. Because the subscales measure related types of ruminative thought, factors were assumed to correlate. In a next step, the two-order model was tested. In the two-order model the loading of one of the five factors on the higher order factor was constrained to 1.0. Factors were not allowed to correlate. Other model specifications remained the same.

To examine goodness of fit of the one and two order model the $\chi^{2}$, a statistic that indicates if a pattern of covariation can be explained by the proposed factor structure, was calculated in each sample. However, because the $\chi^{2}$ is sensitive to sample size, four other indices were calculated as well: (i) the Comparative Fit Index (CFI), (ii) the Tucker-Lewis Index (iii) the Root Mean Square Error of Approximation (RMSEA) and (iv) the Akaike Information Criterion (AIC). Although there is currently little consensus on adequate cut-off scores for fit indices, conventional values are used in this study. CFI and TLI values above $0.90(\mathrm{Hu}$ and Bentler 1999) and values for the RMSEA equal or lower than 0.08 (Browne and Cudeck 1993) are considered to indicate acceptable model fit. The AIC is a measure of relative fit that will be used to compare non-nested models. The model with the lowest AIC value provides the best fit for the data.

The results of the analyses can be found in Table 2 . As can be seen, the correlated one order model (Figs. 1 and 2) provided the best fit of the data on the UGRS in the English sample. Notably, the hierarchical model also showed an appropriate fit on (nearly) all fit indices in the English sample. These findings were corroborated in the Dutch sample, in which the UGRS showed an acceptable model fit for the one order model and the two order model. 
Table 2 Confirmatory factor analyses of the English and Dutch Utrecht Grief Rumination Scale: overall model fit and multi group comparisons

\author{
$\chi^{2}$ Chi square, AIC Aikaike in- \\ formation criterion, $C F I$ compar- \\ ative fit index, TLI Tucker Lewis \\ index, RMSEA root mean square \\ error of approximation \\ Multi group comparisons were \\ used to test the equivalence of \\ the one order factor structure of \\ the English and Dutch version of \\ the UGRS
}

\begin{tabular}{|c|c|c|c|c|c|c|c|c|}
\hline \multirow[t]{2}{*}{ Model } & \multicolumn{5}{|c|}{ Model fit indices } & \multicolumn{3}{|c|}{$\begin{array}{l}\text { Model comparison } \\
\text { tests }\end{array}$} \\
\hline & $\chi^{2}$ & AIC & CFI & TLI & RMSEA & $\Delta \chi^{2}$ & $\Delta d f$ & $p(d)$ \\
\hline \multicolumn{9}{|l|}{ English UGRS } \\
\hline 1. One order model with correlated factors & 192.11 & 272.12 & 0.94 & 0.92 & 0.08 & & & \\
\hline 2. Two order model & 223.76 & 293.76 & 0.92 & 0.90 & 0.09 & & & \\
\hline \multicolumn{9}{|l|}{ Dutch UGRS } \\
\hline 1. One order model with correlated factors & 237.30 & 317.30 & 0.93 & 0.91 & 0.08 & & & \\
\hline 2. Two order model & 266.73 & 336.73 & 0.92 & 0.90 & 0.08 & & & \\
\hline \multicolumn{9}{|c|}{ Multi group comparisons (one order model with correlated factors) } \\
\hline Model 1. Unconstrained model & 448.45 & 608.45 & 0.93 & 0.91 & 0.06 & & & \\
\hline Model 2. Equal factor loadings & 464.87 & 604.90 & 0.93 & 0.91 & 0.06 & & & \\
\hline Model 1 versus Model 2 & & & & & & 16.42 & 10 & 0.09 \\
\hline Model 3. Equal covariances latent variables & 482.66 & 592.65 & 0.93 & 0.92 & 0.06 & & & \\
\hline Model 1 versus Model 3 & & & & & & 17.78 & 15 & 0.27 \\
\hline
\end{tabular}

\section{Multi Group Comparisons}

After conducting separate CFA's for the English and Dutch UGRS, we also tested the cross-cultural equivalence of the UGRS for the one level correlated five factor model, the factor model that provided the best fit. The cross-cultural equivalence was assessed by comparing a model in which the factor loadings and the covariances among the factors were allowed to vary across both language groups (Model 1: Unconstrained model) to a model in which the factor loadings (Model 2: Equal factor loadings) and a model in which the (co)variances among the factors (Model 3: Equal covariances) were constrained to be equal across language groups. To compare the three models, two standard "decrement-to- $\chi^{2}$-test" were used to assess if the overall fit of the models differed (Brown 2006). If a model with more constraints resulted in a significant increase in the overall $\chi^{2}$, indicating a poorer fit, the model with more constraints was rejected in favor of the model with less constraints. Table 2 depicts the results of the multi group comparisons.

First, the unconstrained model (Model 1), which served as a baseline model in the multi group comparisons, was tested. This model produced adequate fit values across both language groups. As before, the $\chi^{2}$-value was significant, but this was disregarded due to the sensitivity of this measure to sample size.

Second, factorial invariance was tested. Factor loadings were constrained to be equal across both samples (Model 2). Fit indices showed that this model also produced a relatively good fit. The difference in $\chi^{2}$-values between Model 1 and Model 2 was not significant, implying that the factor structure of the UGRS was consistent across language groups.

Finally, another set of restrictions was added: (co)variances among the five factors were set to be equal across groups (Model 3). Again, these additional restrictions did not lead to a worse fit, that is, the difference in $\chi^{2}$-values between Model 2 and Model 3 was non-significant. This implies that the associations between the five sub factors were comparable for the English and Dutch versions of the UGRS.

\section{Reliability}

The internal consistency of the total UGRS was excellent in the English sample $(\alpha=0.90)$ and this result was substantiated in the Dutch sample $(\alpha=0.91)$. All subscales of the English UGRS exhibited adequate to good reliability, with $\alpha$ 's of 0.84 (Feelings), 0.88 (Injustice), 0.89 (Counterfactuals), 0.84 (Meaning) and 0.74 (Relationships). The reliability of the subscales of the Dutch UGRS was comparable: with $\alpha$ 's of 0.70 (Feelings), 0.88 (Injustice), 0.90 (Counterfactuals), 0.81 (Meaning) and 0.80 (Relationships). Cronbach's alpha's in the English and Dutch samples were compared using the Alpha test of Hakstian and Whalen (1976). The internal consistencies of the total UGRS and the subscales Injustice, Counterfactuals, Meaning and Relationships did not differ significantly, indicating that these scales show comparable reliability across language groups. However, the Feelings subscale of the English UGRS was more reliable than that of the Dutch version, $M=16.68, p<.001$.

The reliability of the English UGRS was further supported by the item-total correlations. All item-total correlations were high for the English UGRS ( $0.54 \geq r \geq 0.76)$. Furthermore, item scores all correlated positively with the subscales they belonged to. High correlations were found between items and total scores on subscales Reactions $(0.86 \geq r \geq 0.88)$, Injustice $(0.87 \geq r \geq$ $0.92)$, Counterfactuals $(0.89 \geq r \geq 0.92)$, Meaning ( $0.82 \geq r \geq$ $0.92)$ and Relationships $(0.74 \geq r \geq 0.86)$. Again, findings were corroborated in the Dutch sample. Item-total correlations were moderate to high for the Dutch UGRS $(0.49 \geq r \geq 0.72)$ and high correlations were also found between items and total scores on 
Fig. 1 Correlated one order factor structure of the Utrecht Grief Rumination Scale in the English sample

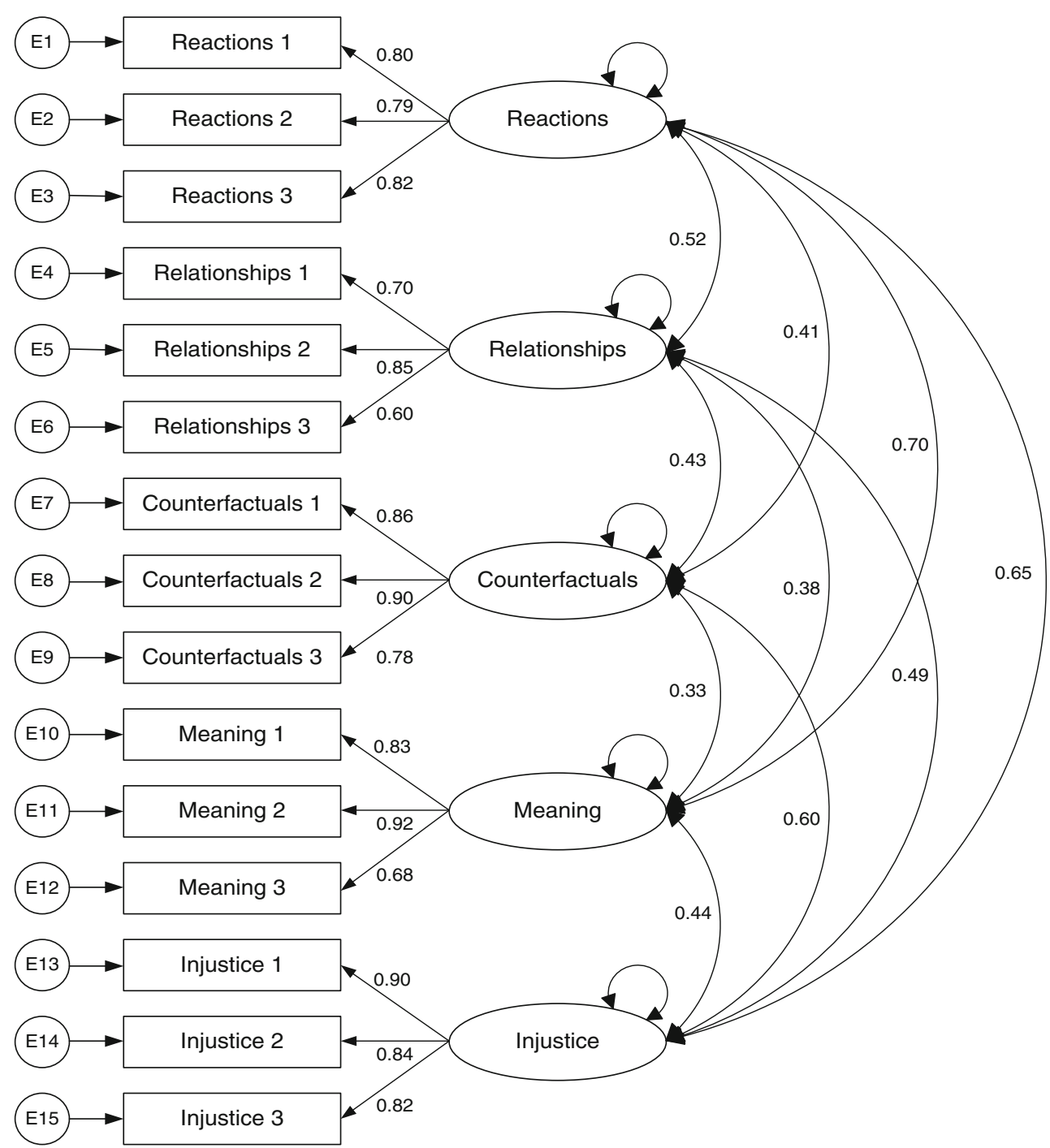

subscales Reactions $(0.76 \geq r \geq 0.81)$, Injustice $(0.87 \geq r \geq 0.91)$, Counterfactuals $(0.87 \geq r \geq 0.94)$, Meaning $(0.76 \geq r \geq 0.88)$ and Relationships $(0.83 \geq r \geq 0.85)$. Fisher Z-tests indicated that item-total correlations did not differ between groups.

\section{Validity}

All correlations between the UGRS and its subscales and other measures relevant to the assessment of validity are shown in Table 3. Notably, Fisher Z-tests indicated that correlations in Table 3 did not differ between language groups. The results of the hierarchical multiple regression analyses conducted to examine the concurrent validity of the UGRS can be found in Tables 4 and 5 .

\section{Convergent Validity}

As predicted, grief rumination correlated positively with other measures of maladaptive ruminative thought; it showed a strong association with brooding measured by the RRS in the English sample $(r=0.54)$. Correlations between the subscales of the UGRS and brooding were all positive and medium to large in size. As predicted, results in the Dutch sample were highly similar. A positive association was also found between grief rumination and trait rumination (RRQ) in the English sample $(r=0.27)$. Furthermore, subscales of the English UGRS showed positive linear associations with trait rumination, with the exception of the subscale Counterfactuals.

\section{Divergent Validity}

In support of the divergent validity of the UGRS, the correlation between grief rumination and a potentially adaptive type of repetitive thought, reflection, was found to be lower than the correlation between grief rumination a maladaptive type of repetitive thought, brooding, in the English sample, $z(200)=$ $3.17, p=.002$. This result was substantiated and in the Dutch sample, $z(313)=7.08, p<.001$. Moreover, in the English 
Fig. 2 Correlated one order factor structure of the Utrecht Grief Rumination Scale in the Dutch sample

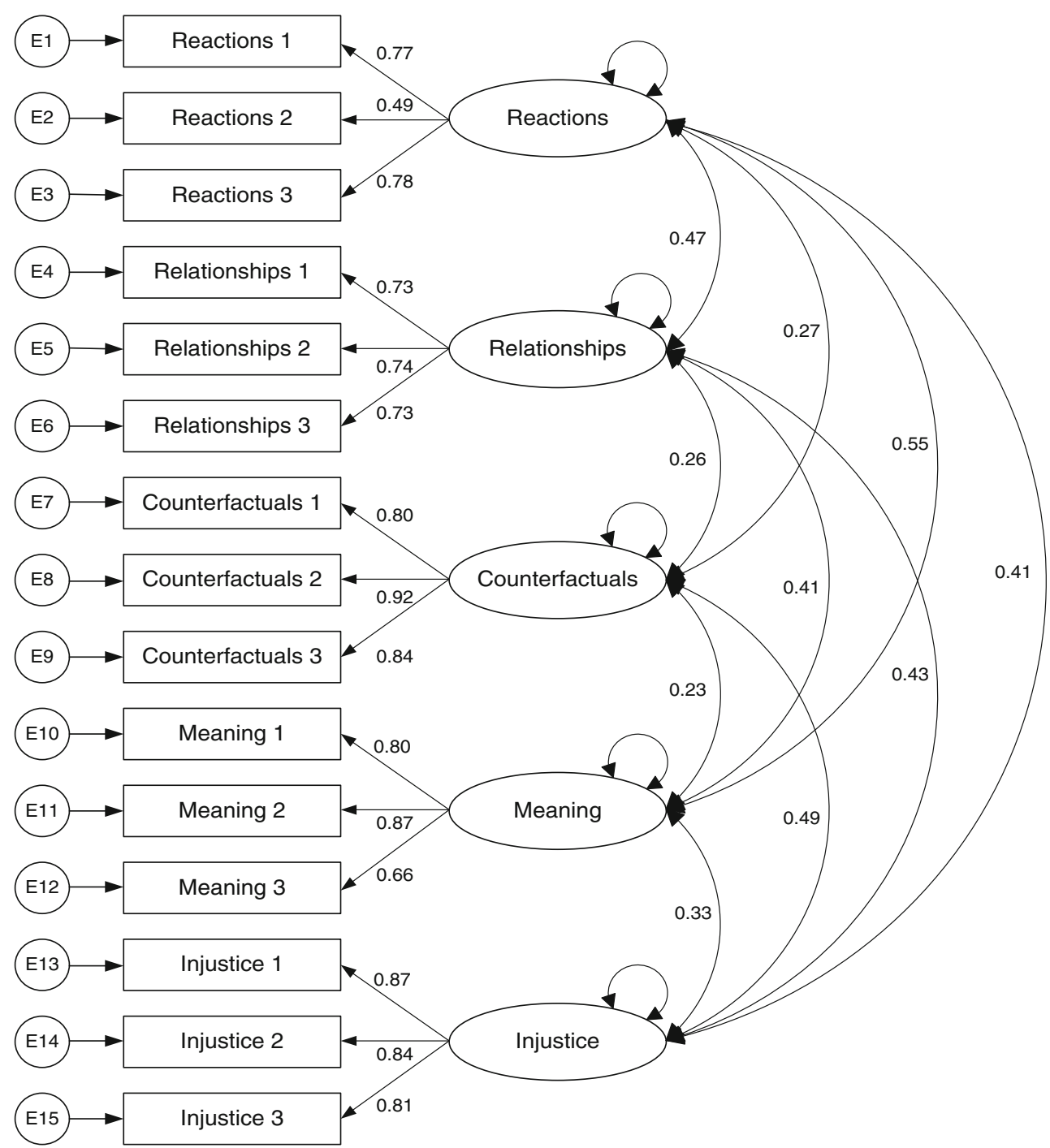

sample, as for the Dutch sample, correlations between subscales of the UGRS and reflection were small to moderate.

\section{Discriminant Validity}

In support of the discriminant validity of the UGRS grief rumination was found to distinguish groups that generally report more mental health problems after the death of a loved one. In the English sample people who had experienced their loss as unexpected on average reported more grief rumination than people who had experienced the death as expected, $t(188)$ $=-3.32, p=.001, d=0.48$. Furthermore, the type of relationship with the deceased was important in determining the level of grief rumination in the English sample, $F(4,198)=3.56$, $p=.008$. Specifically, compared to people who had lost a parent, people who had lost a child, $t(109)=3.34, p=.001$, $d=0.63$, or a partner, $t(155)=2.48, p=.014, d=0.39$, reported more ruminative thoughts. Results in the Dutch sample substantiated these findings. Experiencing an unexpected loss was accompanied by higher levels of grief rumination than the experience of an expected loss, $t(280)=-3.92, p<.001, d=$ 0.47 . Relationship with the deceased was also an important factor for the level of grief rumination in the Dutch group, $F(4,312)=5.06, p<.001$. The parents of a deceased child, $t(124)=3.33, p<.001, \mathrm{~d}=0.59$, and widowers and widows, $t(249)=4.13, p<.001, d=0.52$, ruminated more than people who had lost a parent.

\section{Concurrent Validity}

As can be seen in Tables 4 and 5 , the concurrent validity of the UGRS is supported. Grief rumination concurrently predicted symptom levels of anxiety, depression and complicated grief over and above relevant loss-related variables and brooding, reflection and trait rumination in the English sample. Moreover, the UGRS predicted significant amounts of additional variance in anxious $(4 \%)$, depressive $(5 \%)$ and complicated grief symptoms $(22 \%)$. Grief rumination was a better predictor than 
Table 3 Correlations between UGRS en other constructs in the English and Dutch sample

\begin{tabular}{|c|c|c|c|c|c|c|c|}
\hline & Language & RRS brooding & RRS reflection & RRQ rumination & HADS anxiety & HADS depression & $\mathrm{ICG}$ \\
\hline UGRS & English & 0.54 & 0.35 & 0.27 & 0.53 & 0.45 & 0.74 \\
\hline Total & Dutch & 0.62 & 0.24 & - & 0.48 & 0.49 & 0.73 \\
\hline UGRS & English & 0.46 & 0.41 & 0.27 & 0.44 & 0.34 & 0.54 \\
\hline Reactions & Dutch & 0.33 & 0.43 & - & 0.32 & 0.27 & 0.44 \\
\hline UGRS & English & 0.53 & $0.15^{*}$ & 0.23 & 0.47 & 0.41 & 0.71 \\
\hline Injustice & Dutch & 0.60 & $0.04 n s$ & - & 0.43 & 0.42 & 0.66 \\
\hline UGRS & English & 0.30 & 0.22 & $0.11 n s$ & 0.39 & 0.31 & 0.52 \\
\hline Counterfactuals & Dutch & 0.36 & $0.10 n s$ & - & 0.29 & 0.29 & 0.49 \\
\hline UGRS & English & 0.35 & 0.33 & 0.20 & 0.29 & 0.36 & 0.50 \\
\hline Meaning & Dutch & 0.23 & 0.18 & - & 0.24 & 0.34 & 0.42 \\
\hline UGRS & English & 0.35 & 0.27 & 0.22 & 0.37 & 0.26 & 0.44 \\
\hline Relationships & Dutch & 0.45 & 0.25 & - & 0.35 & 0.37 & 0.48 \\
\hline
\end{tabular}

$U G R S$ Utrecht Grief Rumination Scale, $R R S$ ruminative response scale, $R R Q-R$ rumination subscale of rumination reflection questionnaire, $H A D S$ hospital anxiety and depression scale, $I C G$ inventory of complicated grief, $n s$ not significant

All correlations are significant at $p<0.01$, unless otherwise specified

* Significant at $p<0.05$

other types of ruminative thought for symptoms of complicated grief: the Beta of the UGRS ( $\left.b^{*}=0.58, p<.001\right)$ is much higher than that of brooding $\left(b^{*}=0.23, p<.001\right)$, reflection $\left(b^{*}=-0.01, p>.10\right)$ and rumination measured with the RRQ $\left(b^{*}=0.16, p=.002\right)$. In the Dutch sample these results were corroborated. Grief rumination predicted symptoms of anxiety, depression and complicated grief over and above relevant lossrelated variables, brooding and reflection. Grief rumination was also found to be a significant predictor for anxious, depressive and complicated grief symptoms, explaining $2 \%, 4 \%$ and $12 \%$ additional variance, respectively. Again, grief rumination $\left(b^{*}=0.45, p<.001\right)$ was a better predictor of complicated grief symptoms than brooding $\left(b^{*}=0.39, p<.001\right)$ and reflection $\left(b^{*}=-0.02, p>.10\right)$.

\section{Discussion}

The primary goal of this study was to develop and validate the English version of Utrecht Grief Rumination Scale (UGRS), a self-report scale to measure grief-specific ruminative thought. Concurrently, the psychometric properties of the Dutch version of the UGRS were investigated further. For the English version of the UGRS, as for the Dutch version, confirmatory factor analysis (CFA) showed that a five factor model with five correlated factors (Reactions, Injustice, Counterfactuals, Meaning, Relationships) provided the best fit, when compared to a hierarchical model. An important finding was that a five factor model with one higher-order common factor did not provide the best fit, but the fit was nearly identical to that of the one order model. This supported the claim that the UGRS is a general measure of grief-specific ruminative thought.
Furthermore, multi group comparisons showed that the factor structure of the UGRS was highly similar across both language groups. The model in which the factor loadings and

Table 4 Predictive value of the UGRS for symptoms of psychopathology in the English sample after controlling for relevant loss-related variables and brooding, reflection and rumination (RRQ)

\begin{tabular}{clll}
\hline & Variables & $r^{2}$-change & $\mathrm{b}^{*}$ (final model) \\
\hline Anxiety & & & \\
Block 1 & Loss-related variable & 0.04 & - \\
Block 2 & RRS Brooding & 0.38 & $0.33^{*}$ \\
& RRS reflection & & 0.01 \\
& RRQ rumination & & $0.17^{*}$ \\
Block 3 & UGRS & 0.04 & $0.26^{*}$ \\
Depression & & & \\
Block 1 & RRS brooding & 0.23 & $0.34^{*}$ \\
& RRS reflection & & 0.03 \\
& RRQ rumination & & -0.04 \\
Block 2 & UGRS & 0.05 & $0.26^{*}$ \\
Complicated grief & & \\
Block 1 & Loss-related variables & \\
Block 2 & RRS brooding & 0.07 & - \\
& RRS reflection & & 0.35 \\
Block 3 & RRQ rumination & & -0.01 \\
& UGRS & 0.22 & $0.56^{*}$ \\
\hline
\end{tabular}

$U G R S$ Utrecht Grief Rumination Scale, $R R S$ ruminative response scale, $R R Q$ rumination reflection questionnaire

No loss-related or sociodemographic variables contributed to depressive symptoms. For dummy variables no beta-coefficients are reported

${ }^{\text {a }}$ Sex of the deceased

${ }^{\mathrm{b}}$ Sex of the deceased and relationship with deceased

*Significant at $p<.01$ 
Table 5 Predictive value of the UGRS for symptoms of psychopathology in the Dutch sample after controlling for relevant loss-related variables and brooding and reflection

\begin{tabular}{clll}
\hline & Variables & $r^{2}$-change & $\mathrm{b}^{*}$ (final model) \\
\hline Anxiety & & & \\
Block 1 & RRS brooding & 0.38 & $0.50^{*}$ \\
& RRS reflection & & 0.04 \\
Block 2 & UGRS & 0.02 & $0.16^{*}$ \\
Depression & & & \\
Block 1 & Loss-related variables & \\
Block 2 & RRS brooding & 0.09 & \\
& RRS reflection & 0.20 & $0.31^{*}$ \\
Block 3 & UGRS & 0.04 & -0.02 \\
Complicated grief & & $0.26^{*}$ \\
Block 1 & Loss-related variables & \\
Block 2 & RRS brooding & 0.14 & \\
& RRS reflection & 0.39 & $0.39^{*}$ \\
Block 3 & UGRS & 0.12 & $0.45^{*}$ \\
\hline
\end{tabular}

UGRS Utrecht Grief Rumination Scale, RRS ruminative response scale

No loss-related or sociodemographic variables contributed to anxiety symptoms. For dummy variables no beta-coefficients are reported

${ }^{a}$ Relationship with the deceased

${ }^{\mathrm{b}}$ Relationship with the deceased, cause of death, expectedness of death

*Significant effect at $p<.01$

the covariance structures of English and Dutch UGRS were constrained to be equal produced an acceptable fit. Overall, the series of confirmatory factor analyses supported the construct validity and cross-cultural equivalence of the UGRS in the English and Dutch groups.

The reliability of the UGRS subscales and the total UGRS were also supported and were comparable in both samples. Moreover, earlier preliminary findings on the convergent, divergent, discriminant and concurrent validity of the UGRS (Eisma et al. 2012) were corroborated. All in all, the English and Dutch UGRS showed good psychometric properties.

The reported relationships of grief rumination with other types of repetitive thought and symptoms of psychopathology were highly comparable for the English and Dutch samples. The results showed that grief rumination may best be conceptualized as a maladaptive type of repetitive thinking, because it was more strongly associated with maladaptive components of depressive rumination (i.e., brooding) than with potentially adaptive components of depressive rumination (i.e., reflection) (Treynor et al. 2003). Interestingly, grief rumination was a unique determinant of the outcomes of losing a loved one: it predicts symptoms of anxiety, depression and complicated grief after the loss of a loved one, even when controlling for relevant loss-related and sociodemographic variables and other types of rumination. Notably, grief rumination appeared to be a stronger predictor of complicated grief symptoms than other types of ruminative thinking.
This pattern of results has multiple implications. First, it corroborates the theoretical position that a grief process may be influenced by more than one type of ruminative thinking, as both depressive rumination and grief rumination were significant predictors of symptoms of psychopathology. Second, these two forms of ruminative thinking may be differentially related to persistence and development of different types of psychopathology symptoms. For instance, while grief rumination has been found to be a better predictor of complicated grief symptoms, both concurrently and prospectively (Eisma et al. 2012), brooding is a better concurrent predictor of anxiety symptoms. Third, putting these findings in a broader perspective, results support a differentiated view of various types of repetitive thinking as unique context-specific cognitive styles (e.g., Calmes and Roberts 2007; Rood et al. 2009), rather than subtypes of general repetitive thinking (e.g., McEvoy et al. 2010). Although we acknowledge that identifying generic mechanisms that underlie the effects of most types of repetitive thought is crucial (Watkins 2008; Watkins and Moulds 2012), current results suggest that it is also important to determine for whom, and under what circumstances, specific types of repetitive thinking are adaptive or maladaptive.

Despite the promising results and potential implications of this study, a number of limitations need to be considered. First, all participants were recruited via internet. This implies that some potential participants were excluded from participation in this study. For instance, in 2011, $17 \%$ of UK residents between 16 and 75 years of age (ONS 2011) and $6 \%$ of Dutch adults (CBS 2011) had no internet access. In the UK, people who use the internet generally have a higher income and are less likely to be disabled than people with no internet access (ONS 2011). Consequently, low income groups and people with disabilities may have been underrepresented in the English sample.

Second, we used a voluntary response sample. As a result of self-selection more women than men participated in these studies. According to Stroebe et al. (2001) this stable sex difference may reflect the stronger need for women to express their feelings. However, because a large part of our samples consist of conjugally bereaved individuals, it may also demonstrate the overrepresentation of women in widowhood. Of all women over the age of 65 half is widowed compared with roughly one eighth of men (Arbuckle and de Vries 1995). The generalizability of results across gender may therefore be limited. Notably, in previous research (Eisma et al. 2012) levels of grief rumination did not differ between men and women. This result was replicated in this research in the Dutch sample but not in the English sample; English women ruminated significantly more than English men. Given the small number of men in the English $(n=23)$ and the Dutch group $(n=39)$ additional research is needed to clarify if gender differences in grief-specific rumination exist.

Third, the cross-sectional nature of the current data precludes inferences about the temporal stability and the 
prospective predictive value of the English version of the UGRS. Longitudinal research in a Dutch sample has shown that the UGRS exhibits adequate temporal stability and good predictive validity (Eisma et al. 2012). While psychometric properties of the English and Dutch version of the UGRS show high similarity, additional prospective research is needed to address these issues.

Notwithstanding these limitations, the UGRS is the first validated instrument to measure grief-specific rumination. Based on this study, UGRS appears to be both a reliable and valid measure of grief-specific ruminative thinking. As such, it can be utilized in international scientific research on repetitive thought in bereavement, which is aimed at investigation of the pathways through which emotional problems develop and persist after a loss-experience. Moreover, the UGRS has potential clinical applicability as a brief measure to assess problematic recurrent thought in people who are confronted with bereavement.

\section{References}

Arbuckle, N., \& de Vries, B. (1995). The long term effects of late life spousal and parental bereavement on personal functioning. The Gerontologist, 35, 637-647.

Boelen, P. A., \& van den Hout, M. (2008). The role of threatening misinterpretations and avoidance in emotional problems after loss. Behavioral and Cognitive Psychotherapy, 36, 71-87. doi:10.1017/ S1352465807004079.

Boelen, P. A., van den Bout, J., de Keijser, J., \& Hoijtink, H. (2003a). Reliability and validity of the Dutch version of the Inventory of Traumatic Grief (ITG). Death Studies, 27, 227-247. doi:10.1080/ 07481180302889.

Boelen, P. A., van den Bout, J., \& van den Hout, M. (2003b). The role of negative interpretations of grief reactions in emotional problems after bereavement. Journal of Behavior Therapy and Experimental Psychiatry, 34, 225-238. doi:10.1016/j.jbtep.2003.08.001.

Boelen, P. A., van den Hout, M., \& van den Bout, J. (2006). A cognitivebehavioral conceptualization of complicated grief. Clinical Psychology: Science and Practice, 13, 109-128. doi:10.1111/j. 1468-2850.2006.00013.x.

Bowlby, J. (1980). Attachment and loss: vol 3: Loss, sadness and depression. London: Hogarth Press.

Brown, T. A. (2006). Confirmatory factor analysis for applied research. New York: The Guilford press.

Browne, M. W., \& Cudeck, R. (1993). Alternative ways of assessing model fit. In K. A. Bollen \& J. S. Long (Eds.), Testing structural equation models (pp. 136-162). Beverly Hills, CA: Sage.

Calmes, C. A., \& Roberts, J. E. (2007). Repetitive thought and emotional distress: rumination and worry as prospective predictors of depressive and anxious symptomatology. Cognitive Therapy and Research, 31, 343-356. doi:10.1007/s10608-006-9026-9.

Centraal Bureau voor Statistiek. (2011). Nederland Europees kampioen internettoegang. Retrieved from website Centraal Bureau voor Statistiek: http://www.cbs.nl/nl-NL/menu/themas/vrije-tijd-cultuur/ publicaties/artikelen/archief/2012/2012-3636-wm.htm. Accessed 12 December 2012.

Eisma, M. C., Stroebe, M., Schut, H., Boelen, P. A., van den Bout, J., \& Stroebe, W. (2012). Waarom is dit mij overkomen? Ontwikkeling en validatie van de Utrecht RouwRuminatieSchaal. [Why did this happen to me? Development and validation of the Utrecht Grief Rumination Scale.]. Gedragstherapie, 45, 369-388.

Freud, S. (1917/1957). Mourning and melancholia. In J. Strachey (Ed.), Standard edition of the complete works of Sigmund Freud. London: Hogarth.

Hakstian, A. R., \& Whalen, T. E. (1976). A k-sample significance test for independent alpha coefficients. Psychometrika, 41, 219-231.

Hu, L., \& Bentler, P. M. (1999). Cutoff criteria for fit indices in covariance structure analysis. Conventional criteria versus new alternatives. Structural Equation Modeling, 6, 1-55. doi:10.1080/ 10705519909540118.

Kline, R. B. (2005). Principles and practice of structural equation modeling. New York: Guilford.

Lindemann, E. (1944). Symptomatology and management of acute grief. American Journal of Psychiatry, 101, 141-148.

Martin, L., \& Tesser, A. (1996). Some ruminative thoughts. In R. Wyer (Ed.), Advances in social cognition (Vol. 9, pp. 1-48). Hillsdale, NJ: Lawrence Erlbaum Associates.

McEvoy, P. M., Mahoney, A. E. J., \& Moulds, M. L. (2010). Are worry, rumination and post-event processing one and the same? Development of the repetitive thinking questionnaire. Journal of Anxiety Disorders, 24, 509-519. doi:10.1016/j.janxdis.2010.03.008.

Michael, S. T., \& Snyder, C. R. (2005). Getting unstuck: the role of hope, finding meaning and rumination in the adjustment to bereavement in college students. Death Studies, 29, 435-458. doi:10.1080/ 07481180590932544.

Michael, T., Halligan, S. L., Clark, D. M., \& Ehlers, A. (2007). Rumination in posttraumatic stress disorder. Depression and Anxiety, 24, 307-317. doi:10.1002/da.20228.

Ministerie van Volksgezondheid, Welzijn en Sport. (2002). Wet medisch wetenschappelijk onderzoek met mensen. Retrieved from website centrale commissie voor medisch-wetenschappelijk onderzoek: http://www.ccmo.nl/download/WMO_BRO.pdf.

Nolen-Hoeksema, S. (2001). Ruminative coping and adjustment to bereavement. In M. S. Stroebe, R. O. Hansson, W. Stroebe, \& H. Schut (Eds.), Handbook of bereavement research: Consequences, coping and care (pp. 545-562). Washington DC: American Psychological Association.

Nolen-Hoeksema, S., \& Morrow, J. (1991). A prospective study of depression and posttraumatic stress after a natural disaster: the 1989 Loma Prieta earthquake. Journal of Personality and Social Psychology, 61, 115-121. doi:10.1037//0022-3514.61.1.115.

Nolen-Hoeksema, S., Parker, L. E., \& Larson, J. (1994). Ruminative coping with depressed mood following loss. Journal of Personality and Social Psychology, 67, 92-104. doi:10.1037//0022-3514.67.1.92.

Nolen-Hoeksema, S., McBride, A., \& Larson, J. (1997). Rumination and psychological distress among bereaved partners. Journal of Personality and Social Psychology, 72, 855-862. doi:10.1037// 0022-3514.72.4.855.

Nolen-Hoeksema, S., Wisco, B. E., \& Lyubomirsky, S. (2008). Rethinking rumination. Perspectives on Psychological Science, 3, 400-424. doi:10.1111/j.1745-6924.2008.00088.x.

Office for National Statistics. (2011). Internet access quarterly update. Retrieved from the website Office for National Statistics: http:// www.ons.gov.uk/ons/dcp171766 242415.pdf.

Papageorgiou, W., \& Wells, A. (2003). An empirical test of the clinical metacognitive model of rumination and depression. Cognitive Therapy and Research, 27, 261-273.

Prigerson, H. G., \& Jacobs, S. C. (2001). Traumatic Grief as a distinct disorder: A rationale, consensus criteria and a preliminary empirical test. In M. S. Stroebe, R. O. Hansson, W. Stroebe, \& H. Schut (Eds.), Handbook of bereavement research: Consequences, coping and care (pp. 613-646). Washington DC: American Psychological Association.

Raes, F., Schoofs, H., Hoes, D., Hermans, D., van den Eede, F., \& Franck, E. (2009). Reflection en brooding als subtypes van rumineren: Een 
herziening van de Ruminative Response Scale. [Reflection and brooding as subtypes of rumination: a revision of the Ruminative Response Scale.]. Gedragstherapie, 42, 205-214.

Robinson, M. S., \& Alloy, L. B. (2003). Negative cognitive styles and stress-reactive rumination interact to predict depression: a prospective study. Cognitive Therapy and Research, 27, 275-291.

Rood, L., Roelofs, J., Bögels, S. M., Nolen-Hoeksema, S., \& Schouten, E. (2009). The influence of emotion-focused rumination and distraction on depressive symptoms in non-clinical youth. A metaanalytic review. Clinical Psychology Review, 30, 217-237. doi:10. 1016/j.cpr.2009.07.001.

Spinhoven, P., Ormel, J., Sloekers, P. P. A., Kempen, G. I. J. M., Speckens, A. E. M., \& van Hemert, A. M. (1997). A validation study of the Hospital Anxiety and Depression Scale (HADS) in different groups of Dutch subjects. Psychological Medicine, 67, 363-370. doi:10.1017/S0033291796004382.

Stroebe, M. (1992). Coping with bereavement: a review of the grief work hypothesis. Omega: Journal of Death and Dying, 26, 19-42. doi:10.2190/TB6U-4QQC-HR3M-V9FT.

Stroebe, M., Stroebe, W., \& Schut, H. (2001). Gender differences in bereavement: an empirical and theoretical review. Review of General Psychology, 5, 62-83. doi:10.1037//1089-2680.5.1.62.

Stroebe, M., Schut, H., \& Stroebe, W. (2007). Health outcomes of bereavement. Lancet, 370, 1960-1973. doi:10.1016/S01406736(07)61816-9.

Sukhodolsky, D. G., Golub, A., \& Cromwell, E. N. (2001). Development and validation of the Anger Rumination Scale. Personality and
Individual Differences, 31, 689-700. doi:10.1016/S0191-8869(00) 00171-9.

Tait, R., \& Silver, R. C. (1989). Coming to terms with major negative life events. In J. S. Uleman \& J. A. Bargh (Eds.), Unintended thought (pp. 351-382). New York: Oxford University Press.

Trapnell, P. D., \& Campbell, J. D. (1999). Private self-consciousness and the five-factor model of personality: Distinguishing rumination from reflection. Journal of Personality and Social Psychology, 76, 284 304. doi:10.1037//0022-3514.76.2.284.

Treynor, W., Gonzalez, J., \& Nolen-Hoeksema, S. (2003). Rumination reconsidered: a psychometric analysis. Cognitive Therapy and Research, 27, 247-259.

van der Houwen, K., Stroebe, M., Schut, H., Stroebe, W., \& van den Bout, J. (2010). The mediating processes of bereavement: The role of rumination, threatening grief interpretations and deliberate grief avoidance. Social Science and Medicine, 71, 1669-1676. doi:10. 1016/j.socscimed.2010.06.047.

Watkins, E. (2008). Constructive and unconstructive repetitive thought Psychological Bulletin, 134, 163-206. doi:10.1037/0033-2909.134. 2.163.

Watkins, E. R., \& Moulds, M. L. (2012). Repetitive thought: Rumination in complicated grief. In M. S. Stroebe, H. Schut, \& J. van den Bout (Eds.), Complicated grief: Scientific foundations for health care professionals (pp. 163-175). New York: Routledge.

Zigmond, A. S., \& Snaith, R. P. (1983). The Hospital Anxiety and Depression Scale. Acta Psychiatrica Scandinavica, 67, 361-370. doi:10.1111/j.1600-0447.1983.tb09716.x. 